\title{
Avaliação das perdas em um sistema de abastecimento de água de pequeno porte no semiárido brasileiro por aspectos multicriteriais
}

\author{
Evaluation of losses in small water supply systems in the \\ Brazilian semiarid by multicriteria aspects
}

Cinthia Maria de Abreu Claudino ${ }^{1}$ (D), Vanine Elane Menezes de Farias ${ }^{2}$ (D), Alessandra Gomes Brandão ${ }^{3}$ (D), Igor Souza Ogata ${ }^{4}$ (D), Rivaildo da Silva Ramos Filho ${ }^{5}$ (D), Thiago de Sá Sena ${ }^{5}$ (D)

${ }^{1}$ Universidade Federal da Paraíba - UFPB, João Pessoa, PB, Brasil. E-mail: cinthiamariaac@gmail.com 2Universidade Federal de Pernambuco - UFPE, João Pessoa, PB, Brasil. E-mail: vaninemenezesf@gmail.com ${ }^{3}$ Universidade Estadual da Paraíba - UEPB, Araruna, PB, Brasil. Email: alessandra.gomes.brandao@gmail.com ${ }^{4}$ Universidade Estadual da Paraíba - UEPB, Campina Grande, PB, Brasil. E-mail: igor.ogata@gmail.com

${ }^{5}$ Universidade Federal de Campina Grande - UFCG, Campina Grande, PB, Brasil. E-mails: rivaildofilho31@gmail.com, engthiagosena@gmail.com

\begin{abstract}
Como citar: Claudino, C. M. A., Farias, V. E. M., Brandão, A. G., Ogata, I. S., Ramos Fiho, R. S., \& Sena, T. S. (2021). Avaliação das perdas em um sistema de abastecimento de água de pequeno porte no semiárido brasileiro por aspectos multicriteriais. Revista de Gestão de Água da América Latina, 18, e13. https://doi.org/10.21168/rega.v18e13
\end{abstract}

\begin{abstract}
RESUMO: Diante da crise hídrica que assola a região semiárida brasileira, a busca por alternativas que forneçam água em quantidade e qualidade adequada tem se tornado relevante. Neste cenário, uma investigação sobre as perdas de água é de fundamental importância no processo de gestão da demanda, por esta representar um problema frequente em sistemas de abastecimento de água que, especificamente no semiárido, pode consumir valores acima de $85 \%$ da água produzida. À vista disso, este trabalho selecionou como objeto de estudo o sistema de abastecimento de água do município de Araruna - PB, a fim de definir uma forma de determinar as ações prioritárias para minimizar as perdas em um sistema de abastecimento de água, utilizando aspectos multicriteriais. Para tanto, o método ELECTRE II foi alimentado por indicadores do Sistema Nacional de Informações sobre Saneamento que representem perdas segundo critérios econômicos, sociais e ambientais. Concluiu-se que a gestão das perdas é mais influenciada pelos critérios sociais, devendo ser o foco das ações prioritárias. Contudo, os demais critérios não apresentaram resultados adequados e também requerem atenção. A vista disso, é importante ressaltar a capacidade do método em auxiliar tomadores de decisão na seleção de ações prioritárias que possam apresentar resultados direcionados a soluções referentes ao atendimento total de água e ao volume de água disponibilizado por economia no combate as perdas de sistemas de abastecimento de água.
\end{abstract}

Palavras-chave: Perdas de Água; Análise Multicriterial; ELECTRE II; Indicadores de Desempenho.

ABSTRACT: In the midst of the water crisis that is plaguing the Brazilian semiarid region, the search for alternatives that provide water in adequate quantity and quality has become relevant. In this scenario, research on water losses is fundamental in the demand management process, as this represents a frequent problem in water supply systems, which specifically in the semiarid region can consume values above $85 \%$ of the water produced. Therefore, this work selected the water supply system in the city of Araruna - PB, in order to define a way that determines priority actions to minimize losses in water supply systems, using multicriteria aspects. To this end, the ELECTRE II method was powered by indicators from the National Sanitation Information System that represent losses according to economic, social and environmental criteria. Thus, it was concluded that loss management is more influenced by social criteria and should be the focus of priority actions. However, the other criteria did not show adequate results and also need attention. Therefore, it is important to emphasize the method's ability to assist decision makers in the selection of priority actions that can present results aimed at solutions related to total water service and the volume of water made available by savings in combating the losses of water supply systems.

Keywords: Water Losses; Multicriterial Analysis; ELECTRE II; Performance Index.

Recebido: Maio 19, 2021. Revisado: Julho 28, 2021. Aceito: Agosto 24, 2021. 


\section{INTRODUÇÃO}

As regiões semiáridas brasileiras se caracterizam por seu balanço hídrico deficitário, em que a precipitação média anual é menor que a evaporação potencial anual da região. A demanda por água nessas regiões vem aumentando constantemente devido ao crescimento populacional, dos níveis de urbanização e industrialização, assim como pelo aumento da produção e do consumo (Nunes et al., 2020). Integrada a escassez hídrica crônica gerada pelo clima e aumento da demanda hídrica, existe, ainda, uma estruturação fundiária que beneficia os grandes proprietários de terra e a ineficiência do estado em promover políticas públicas eficazes para a conservação dos recursos hídricos (Albuquerque et al., 2019).

Associado a isso, muitos municípios situados em regiões semiáridas apresentam problemas em seus Sistemas de Abastecimento de Água - SSA, devido à fatores como extensos períodos de estiagem que, ao diminuir a disponibilidade de água bruta, causam intermitência no abastecimento. Em decorrência dessa intermitência, os sistemas de abastecimento de água dos municípios ficam expostos a perturbações e estresses, tornando-os mais sensíveis e gerando, assim, vulnerabilidade para os seus usuários (Turner et al., 2003; Adger, 2006; Boruff et al., 2018).

Para resolução dessa problemática, de maneira a encontrar alternativas para disponibilizar água em quantidade e qualidade adequada ao desenvolvimento humano, tradicionalmente é realizado o aumento da oferta, instalando grandes obras hídricas como construção de reservatórios, perfuração de poços e transposição de águas. No entanto, em muitos casos, essas ações não são viáveis do ponto de vista econômico, financeiro e ambiental (Gnadlinger, 2015).

Frente às limitações da ampliação da oferta de água, uma alternativa a ser utilizada para solução do problema é a gestão da demanda. A necessidade de uma solução alternativa como essa é mais explícita nas regiões áridas e semiáridas, onde o clima, a obsolescência dos SAA e a defeituosa e/ou ausência de gestão dos recursos hídricos geram graves problemas para a população (Guedes et al., 2014). Neste sentido, uma forma de aumentar a eficiência do uso da água está associada ao controle de perdas nos sistemas de distribuição. Essa perda, provocada pelos vazamentos ao longo da rede, acarretam em maior consumo de energia e perdas econômicas (Colombo \& Karney, 2005; Gupta et al. 2017), em maior ônus financeiros para os consumidores, além de propiciar a contaminação da água (Kouchi et al., 2017).

Analisando o aspecto de perdas nos sistemas de distribuição no Brasil, de acordo com o Sistema Nacional de Informações sobre Saneamento (SNIS), as empresas prestadoras de serviços de abastecimento comumente operam com índices de perdas na distribuição que variam entre 30\% e 60\% e, no semiárido nordestino - onde a escassez hídrica é severa - existem municípios que alcançam mais de 85\% (Brasil, 2018). Nessa perspectiva, é possível verificar a necessidade de ações voltadas ao controle de perdas para solução dos problemas de disponibilidade de água, realizando medidas preventivas e reparadoras através de métodos técnicos e probabilísticos que consigam interligar a realidade do local à técnica mais eficiente, a fim de que sejam obtidos os resultados de diminuição das perdas com a maior efetividade possível.

Biasutti \& Coelho (2019) apontam como os prestadores do serviço de abastecimento têm como desafio desenvolver e implementar indicadores capazes de avaliar a eficácia na qualidade do serviço prestado e a eficiência. Segundo o Instituto Trata Brasil (2015), as perdas são um ponto frágil dos sistemas de saneamento e das empresas responsáveis pela operação do serviço, sejam públicas ou privadas e os dados atuais mostram a fragilidade da gestão de grande parte do setor.

Devido a área de gestão dos recursos hídricos envolver interesses múltiplos e conflitantes, haja vista a variedade de atores e agentes envolvidos, é necessário trabalhar com uma metodologia própria para a gestão e planejamento desses recursos (Pompermayer et al., 2007). Sendo assim, a análise multicritério se torna uma ferramenta importante nesse processo, pois permite o auxílio à tomada de decisão quando o problema abordado envolve uma diversidade de critérios.

Diante disso, esse estudo buscou estabelecer o cenário atual de perdas de um sistema de abastecimento de água na microrregião do Curimataú Oriental no estado da Paraíba, a fim de determinar as ações prioritárias para minimizar as perdas, utilizando aspectos multicriteriais. Para isso, foram feitas análises nas séries históricas do Î́ndice de perdas de Faturamento (IN013), do Índice de perdas na distribuição (IN049), do Índice de perdas por ligação (IN051) e do Índice bruto de perdas lineares (IN050). Assim como a análise multicritério em um SAA de pequeno porte, que sofre com constantes períodos de racionamento.

\section{METODOLOGIA}

O processo metodológico consistiu em caracterizar o Sistema de Abastecimento de Água do município de Araruna - PB, localização na Figura 1, descrevendo suas condições de infraestrutura e operação, focando esta última na sua condição de perdas. Subsequentemente, indicadores foram selecionados para compor critérios que determinem a condição econômica, social e ambiental do SAA 
e forneça dados para a análise multicriterial a ser posteriormente utilizada, de forma a apoiar uma tomada de decisão racional e adequada aos objetivos propostos.

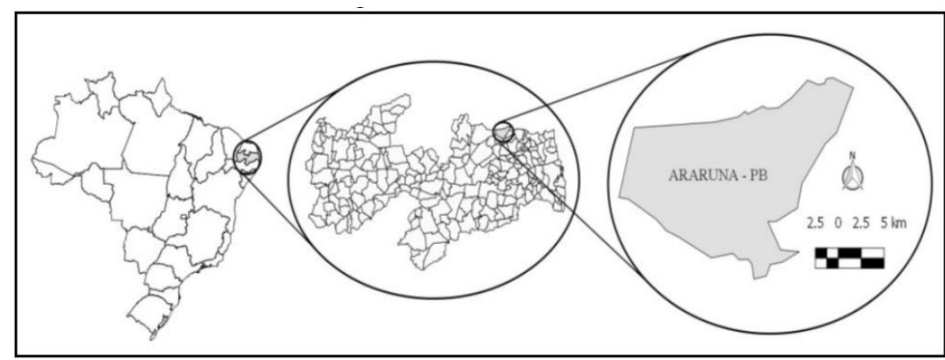

Figura 1 - Localização geográfica do município de Araruna - PB. Fonte: Neves (2016)

\subsection{Caracterização do sistema}

A caracterização da infraestrutura do SAA do município de Araruna - PB foi realizada a partir de um questionário enviado à Companhia de Água e Esgotos da Paraíba (CAGEPA), de maneira a conhecer todas as unidades que compõem o sistema, desde o manancial até a distribuição. As perguntas do questionário solicitaram informações a respeito dos aspectos estruturais do sistema de distribuição, tais como: Qual a quantidade e a tipologia das unidades existentes no sistema de distribuição? Qual o tipo de material empregado no processo construtivo do sistema? Quais os valores de volumes micromedidos e macromedidos? e Qual a política tarifária empregada?

Além da infraestrutura, buscou-se estudar o modelo operacional do sistema, com ênfase nas perdas ocorridas no processo de fornecimento de água. Para isso, informações referentes a esse aspecto foram retiradas do SNIS, o sistema de informação nacional que fornece dados sobre todos os sistemas de abastecimento de água brasileiros, seja público ou privado. Neste sentido, foram analisados historicamente os dados de perdas reais, também denominada de perdas na distribuição pelo SNIS, as perdas aparentes, perdas totais, perdas de faturamento, perdas lineares e perdas por ligação.

\subsection{Indicadores de perdas do Sistema Nacional de Informações sobre Saneamento}

Dentro da plataforma SNIS existem 4 índices que tratam sobre a perda de água dentro de um SAA, denominados de índice de perdas na distribuição, índice de perdas de faturamento, índice bruto de perdas lineares e índice de perdas por ligação, conforme descritos no Quadro 1.

Quadro 1 - Índices de perdas de água no padrão do SNIS

\begin{tabular}{|c|c|c|}
\hline Índices & Formas de cálculo & $\begin{array}{l}\text { Significado das } \\
\text { informações }\end{array}$ \\
\hline $\begin{array}{c}\text { Perdas na } \\
\text { distribuição (\%) }\end{array}$ & $\frac{A \mathbf{G} 006+\mathbf{A G} 018-\mathbf{A G} 010-\mathbf{A G} 024}{\mathbf{A G} 006+\mathbf{A G} 018-\mathbf{A G} 024}$ X100 & $\begin{array}{l}\text { AG002: Quantidade de } \\
\text { ligações ativas de água; }\end{array}$ \\
\hline $\begin{array}{c}\text { Perdas de } \\
\text { faturamento (\%) }\end{array}$ & $\frac{\mathbf{A G} 006+\text { AG018- AG011- AG024 }}{\text { AG006+ AG018-AG024 }}$ X100 & $\begin{array}{l}\text { AG005: Extensão da rede } \\
\text { de água; } A G 006: \text { Volume } \\
\text { de água produzido: }\end{array}$ \\
\hline $\begin{array}{c}\text { Índice bruto de } \\
\text { perdas lineares } \\
\left(\mathrm{m}^{3} / \mathrm{dia} / \mathrm{km}\right) \\
\end{array}$ & $\frac{\mathbf{A G} 006+\mathbf{A G} 018-\mathbf{A G} 010-\mathbf{A G} 024}{\mathbf{A G} 005} \times \frac{1.000}{365}$ & $\begin{array}{l}\text { AG010: Volume de água } \\
\text { consumido; } A G 011: \\
\text { Volume de água faturado; }\end{array}$ \\
\hline $\begin{array}{l}\text { Perdas por ligação } \\
\text { (l/dia/ligações) }\end{array}$ & $\frac{\mathbf{A G} 006+\mathbf{A G} 018-\mathbf{A G} 010-\mathbf{A G} 024}{\mathbf{A G} 002} \times \frac{1.000 .000}{365}$ & $\begin{array}{l}\text { AG018: Volume de água } \\
\text { tratada importado; } \\
\text { AG024: Volume de } \\
\text { serviço. }\end{array}$ \\
\hline
\end{tabular}

\subsubsection{Seleção dos indicadores}

Os indicadores de perdas foram adotados segundo o Glossários de Informações e Indicadores, no endereço eletrônico do SNIS (www.snis.gov.br/glossarios), que é, atualmente, a base de dados mais completa sobre o setor de saneamento brasileiro. As siglas IN e FN que compõem o código do glóssario 
do SNIS indicam ao que se relaciona os indicadores. A siglas com FN estão relacionadas aos dados financeiros, como despesas e receita. Já siglas com IN estão ligadas a índices utilizados pelo SNIS.

Foram selecionados 10 indicadores (Quadro 2), organizados no âmbito econômico, social e ambiental, representando os aspectos do sistema de abastecimento que podem influenciar no processo de perda de água no sistema. A organização dos indicadores dentro dessas três categorias foi baseada nas discussões feitas por Albuquerque et al. (2008), onde a forma como a categorização foi estabelecida possui relação com fatores que direta ou indiretamente possam causar perdas no sistema. 0 aspecto econômico, por exemplo, que abrange o desempenho financeiro, a evasão de receitas, a produtividade, o faturamento e os investimentos, influencia nas perdas do sistema, pois o desempenho econômico está diretamente ligado as melhorias e manutenções feitas no SAA que garantem o adequado funcionamento e consequentemente a diminuição das perdas.

Quadro 2 - Descrição dos indicadores utilizados

\begin{tabular}{|c|c|c|}
\hline Categoria & Indicadores e código SNIS & Descrição \\
\hline \multirow{5}{*}{ Econômica } & $\begin{array}{l}\text { E1 - Indicador de desempenho financeiro } \\
\text { (IN012) }\end{array}$ & $\begin{array}{c}\text { Representa a razão entre a receita operacional e as } \\
\text { despesas da prestação do serviço }\end{array}$ \\
\hline & E2 - Índice de evasão de receitas (IN029) & $\begin{array}{c}\text { Representa a dívida dos usuários com a companhia, } \\
\text { com base na receita total e a arrecadação total }\end{array}$ \\
\hline & E3 - Índice de produtividade (IN045) & $\begin{array}{c}\text { Representa a eficiência da prestação do serviço pela } \\
\text { razão entre a quantidade de empregados e } \\
\text { quantidade de ligações ativas }\end{array}$ \\
\hline & E4 - Índice de faturamento de água (IN028) & $\begin{array}{l}\text { Representa o volume de água que foi faturado, em } \\
\text { relação ao volume total de água utilizado no sistema }\end{array}$ \\
\hline & $\begin{array}{l}\text { E5 - Índice de investimentos no sistema de } \\
\text { água (FN023+FN042+FN052)/FN005 }\end{array}$ & $\begin{array}{c}\text { Representa a quantidade de recursos financeiros } \\
\text { que foi investido no sistema de abastecimento de } \\
\text { água em relação a receita obtida }\end{array}$ \\
\hline \multirow{2}{*}{ Social } & $\begin{array}{l}\text { S1 - Índice de atendimento total de água } \\
\text { (IN055) }\end{array}$ & $\begin{array}{l}\text { Representa a razão entre a população atendida com } \\
\text { abastecimento de água e a população total }\end{array}$ \\
\hline & $\begin{array}{l}\text { S2 - Volume de água disponibilizado por } \\
\text { economia (IN025) }\end{array}$ & $\begin{array}{l}\text { Representa o volume de água fornecido para cada } \\
\text { economia ativa do sistema de abastecimento }\end{array}$ \\
\hline \multirow{3}{*}{ Ambiental } & $\begin{array}{c}\text { A1 - Consumo médio percapita de água } \\
\text { (IN022) }\end{array}$ & $\begin{array}{c}\text { Representa a quantidade média que cada pessoa } \\
\text { consome de água }\end{array}$ \\
\hline & $\begin{array}{l}\text { A2 - Índice de micromedição relativo ao } \\
\text { consumo (IN044) }\end{array}$ & $\begin{array}{l}\text { Representa a quantidade de água que é } \\
\text { micromedida em relação a toda água consumida }\end{array}$ \\
\hline & A3 - Índice de macromedição (IN011) & $\begin{array}{l}\text { Representa a quantidade de água macromedida em } \\
\text { relação a toda água utilizada no sistema }\end{array}$ \\
\hline
\end{tabular}

O âmbito social, que engloba o índice de atendimento e a disponibilidade de água por economia, expressa o cenário de abrangência e suficiência do sistema e está, dessa forma, diretamente ligada as perdas, uma vez que quanto maior o atendimento e volume de água maior a chance de o sistema apresentar perdas significativas.

Por outro lado, o fator ambiental foi levado em consideração devido aos impactos ambientais causados por um SAA em condições indevidas, como situações que afetem a operação do sistema e gera desperdícios, como por exemplo vazamentos, ligações candestinas e outros defeitos que demadem além do valor para o qual aquele sistema foi projetado.

Dessa forma, os índices selecionados foram verificados separadamente da seguinte forma: a) se o consumo médio per capita for acima do estimado ocorre desperdício e maior chance de perdas; b) o índice de macromedição representa o volume de água para grandes vazões no processo de captação de água bruta, capaz de indicar perdas nas grandes unidades dos sistemas de abastecimento; e c) o índice de micromedição, que mede a quantidade de água fornecida a uma economia pela rede distribuição, fornecendo assim o total de perdas no sistema de distribuição para ser comparado com o índice de macromedições.

\subsection{Seleção de Método Multicritério de Apoio a Decisão}

De acordo com Gomes Júnior et al. (2008), a existência de mais de um ponto de vista a ser considerado para a comparação entre um grupo de alternativas e a determinação da 
superioridade de uma sobre a outra pode ser bastante complexo, o que leva a necessidade da utilização de ferramentas capazes de resolver este tipo de questão. Os métodos multicritério ajudam a solucionar problemas complexos de decisão, considerando os diferentes pontos de vistas, de forma a analisar o desempenho de cada alternativa com relação ao objetivo proposto. Segundo Mota \& Almeida (2007), esses métodos podem ser classificados a partir de diferentes abordagens: a) abordagem do critério único de síntese, que agrega diferentes pontos de vista dentro de uma única função de síntese, que pode ser posteriormente otimizada; b) abordagem da sobreclassificação, que apoia, em primeiro lugar, a construção de uma relação de sobreclassificação, que representa as preferências estabelecidas pelo decisor e depois consiste em explorar a relação de sobreclassificação de tal forma que ajude o decisor a resolver o seu problema; c) abordagem do julgamento interativo, que são métodos que utilizam a abordagem de tentativas e erros e estruturas de programação matemática multiobjetivo.

\subsubsection{Método ELECTRE II}

Os métodos Elimination and (et) choice Translating Reality Algorithm - ELECTRE são classificados como um método multicriterial de sobreclassificação, ou seja, baseiam-se na construção de uma relação de sobreclassificação que incorpora as preferências estabelecidas pelo decisor diante das alternativas disponíveis (Venturini, 2003). Também denominado de métodos de subordinação ou prevalência, é composto pelos métodos ELECTRE I, II, III, IV, IS e TRI (Szajubok et al., 2006), no qual para cada problema existe uma versão do ELECTRE mais adequada a ser utilizada.

O método selecionado para a aplicação foi o ELECTRE II devido a vasta aplicação em trabalhos científicos para avaliar diferentes sistemas produtivos (Ishizaka \& Nemery, 2013; Silva, 2019; Gomes Júnior et al., 2008), logo essa robustez pode trazer uma aplicação adequada para SAA. Dentre os métodos ELECTRE, este é o que melhor se adequa ao problema de decisão em questão por se tratar de critérios verdadeiros, visto que para a problemática se ordenam as alternativas da melhor para a pior, isto é, ordena a escolha, assim é o método mais aplicável (Govindan \& Jepsen, 2016).

\subsection{Aplicação do método de análise}

Para fundamentar o processo de tomada de decisão o método ELECTRE II foi aplicado em duas fases. A primeira fase consistiu em uma comparação par a par entre as alternativas, fazendo assim uma construção de preferência e não preferência, com vista em categorias tidas como essenciais para caracterização dos sistemas de água, como operacional, econômico e ambiental. Posteriormente, na segunda fase, é aplicado o algoritmo em que a função problema construída é resolvida, resultando na sobreclassificação (Oliveira et al., 2013).

\subsubsection{Normalização dos indicadores}

Para que o método seja desenvolvido foi necessário realizar a etapa de normalização. Essa é uma etapa importante no processo, pois os indicadores selecionados possuem multiplicidade de unidades e não seria metodologicamente adequado trabalhar e comparar indicadores nesta situação, portanto estes foram transformados em valores adimensionais.

Para normalização, foram utilizados limites superiores e inferiores, sendo esses limites definidos, respectivamente, como o melhor e o pior desempenho dos índices no campo de análise das cinco regiões do país para o ano de referência de 2018 - ano mais atual disponibilizado pela base de dados do SNIS. 0 cálculo dos índices de cada região foi realizado através da média do valor do índice de cada município da região, sendo para região centro-oeste calculada a média para 467 municípios, na região Nordeste 1794 municípios, na região Norte 450 municípios, na região Sudeste 1668 municípios e na região Sul 1191 municípios. Os limites definidos estão expostos no Quadro 3. 
Quadro 3 - Limites para normalização

\begin{tabular}{|c|c|c|c|c|c|}
\hline & \multicolumn{5}{|c|}{ Indicadores } \\
\hline $\begin{array}{c}\text { Regiões } \\
\text { brasileiras }\end{array}$ & $\begin{array}{c}\text { E1 - Indicador de } \\
\text { desempenho financeiro }\end{array}$ & $\begin{array}{c}\text { E2 - Índice de } \\
\text { evasão de } \\
\text { receitas }\end{array}$ & $\begin{array}{l}\text { E3 - Índice de } \\
\text { produtividade }\end{array}$ & $\begin{array}{c}\text { E4 - Índice de } \\
\text { faturamento de } \\
\text { água } \\
\end{array}$ & $\begin{array}{l}\text { E5 - Índice de } \\
\text { investimento }\end{array}$ \\
\hline Centro-Oeste & 105,61 & 1,14 & 3,36 & 73,65 & 0,09 \\
\hline Nordeste & 88,89 & 12,28 & 2,74 & 71,12 & 54,40 \\
\hline Norte & 124,72 & 21,45 & 5,20 & 42,89 & 0,32 \\
\hline Sudeste & 96,91 & 3,09 & 3,49 & 75,03 & 0,16 \\
\hline Sul & 97,78 & 1,58 & 3,05 & 74,22 & 0,11 \\
\hline Maior & 124,72 & 21,45 & 5,20 & 75,03 & 54,40 \\
\hline Menor & 88,89 & 1,14 & 2,74 & 42,89 & 0,09 \\
\hline $\begin{array}{c}\text { Regiões } \\
\text { brasileiras }\end{array}$ & $\begin{array}{c}\text { S1 - Índice de } \\
\text { atendimento total de } \\
\text { água }\end{array}$ & $\begin{array}{c}\text { S2 - Volume de } \\
\text { água } \\
\text { disponibilizado } \\
\text { por economia } \\
\end{array}$ & $\begin{array}{c}\text { A1 - Consumo } \\
\text { médio per capita } \\
\text { de água }\end{array}$ & $\begin{array}{l}\text { A2 - Índice de } \\
\text { micromedição } \\
\text { relativo ao } \\
\text { consumo } \\
\end{array}$ & $\begin{array}{l}\text { A3 - Índice de } \\
\text { macromedição }\end{array}$ \\
\hline Centro-Oeste & 74,79 & 16,67 & 156,52 & 91,01 & 81,30 \\
\hline Nordeste & 62,07 & 18,60 & 103,33 & 79,37 & 49,36 \\
\hline Norte & 52,78 & 31,14 & 132,06 & 50,02 & 10,75 \\
\hline Sudeste & 75,51 & 17,24 & 164,15 & 90,13 & 76,36 \\
\hline Sul & 75,65 & 15,71 & 138,90 & 94,25 & 58,08 \\
\hline Maior & 75,65 & 31,14 & 164,15 & 94,25 & 81,30 \\
\hline Menor & 52,78 & 15,71 & 103,33 & 50,02 & 10,75 \\
\hline
\end{tabular}

Além da definição dos limites, o processo de normalização é feito com a utilização de equações. Para a pesquisa foi escolhido o método normativo de redimensionamento contínuo que classifica os valores entre 0 e 1 através da utilização das Equações 1 e 2, sendo a primeira aplicada para o caso em que o índice apresenta uma melhor eficiência quanto maior o seu valor. Para os casos contrários em que os índices são mais eficientes quanto menor seu valor, utiliza-se a segunda equação.

$S_{i}=\frac{X_{i}-X_{i n f}}{X_{\text {sup }}-X_{i n f}}$

$S_{i}=1-\left(\frac{X_{i}-X_{i n f}}{X_{\text {sup }}-X_{i n f}}\right)$

Onde:

$S_{i}=$ valor normalizado.

$X_{i}=$ valor a ser normalizado.

$X_{\text {inf }}=$ limite inferior.

$X_{\text {sup }}=$ limite superior.

No caso em que o valor normalizado for menor que 0 , será adotado como 0 , assim como, para o caso do valor normalizado ser maior que 1, será adotado a unidade.

Neste trabalho, o método passou por uma modificação, em que devido a necessidade de um limite ótimo para o consumo médio de água, esse valor ideal é de $100 \mathrm{~L} /$ hab.dia e os limites superior e inferior de $200 \mathrm{~L} /$ hab.dia e $50 \mathrm{~L} /$ hab.dia, respectivamente (Ogata, 2014). A Equação 3 foi aplicada no caso do consumo que está entre 50 l/hab./dia e 100 l/hab./dia, já a Equação 4 foi aplicada nos casos do consumo está entre 100 l/hab./dia e 200 l/hab./dia.

$S_{i}=\frac{X_{i}-50[1 / \text { hab. } / \text { dia }]}{100-50}$

$S_{i}=1-\left(\frac{X_{i}-100[1 / \mathrm{hab} . / \mathrm{dia}]}{200-100}\right)$

Onde: 
$\mathrm{S}_{\mathrm{i}}=$ valor normalizado.

$\mathrm{X}_{\mathrm{i}}=$ valor a ser normalizado.

No caso em que o consumo per capita de água for menor que $50 \mathrm{l} / \mathrm{hab}$./dia ou maior 200 l/hab./dia, o valor normalizado é 0.

\subsubsection{Ponderação}

A outra etapa necessária para aplicação do método ELECTRE II diz respeito a definição dos pesos, com intuito de realçar a importância de cada indicador para a eficiência do sistema de saneamento básico.

Como o objetivo da aplicação do método na pesquisa foi a resolução de qual a categoria mais deficiente e que implica em maiores perdas de água para o sistema analisado, optou-se por realizar uma ponderação baseada nos problemas mais recorrentes ligados as perdas (reais e aparente), como vazamentos, ligações clandestinas e erros de leitura, identificados a partir do estudo de perdas de água do Instituto Trata Brasil (2018) com dados do ano de 2018 do SNIS e de 2003 do Ministério da Cidades.

A escala de ponderação foi baseada na utilizada por Albuquerque et al. (2008), que montou uma hierarquia diferenciando a influência dentre todos indicadores de modo a compreender globalmente a interação entre os dados avaliados. Assim, a ponderação se configurou de forma adaptada como sendo 1,0 para quando a relação dos índices com a causa de perdas era muito alta, 0,8 em que a relação era alta, 0,6 em que a relação era média, 0,4 com a relação baixa e 0,2 relação muito baixa. Como todos os índices foram selecionados devido sua relação com os problemas de perdas o mínimo utilizado na escala de ponderação foi de 0,6. A ponderação de cada índice foi exposta no Quadro 4.

Quadro 4 - Valores de ponderação para os indicadores selecionados

\begin{tabular}{c|c}
\hline \multicolumn{1}{c}{ Indicadores } & Ponderação \\
\hline E1- Indicador de desempenho financeiro & 0,6 \\
\hline E2 - Índice de evasão de receitas & 0,6 \\
\hline E3 - Índice de produtividade: & 1,0 \\
\hline E4- Índice de faturamento de água & 0,8 \\
\hline E5-Índice de investimento & 1,0 \\
\hline S1 - Índice de atendimento total de água & 1,0 \\
\hline S2 - Volume de água disponibilizado por economia & 0,6 \\
\hline A1 - Consumo médio per capita de água & 0,6 \\
\hline A2- Índice de micromedição relativo ao consumo & 1,0 \\
\hline A3- Índice de macromedição & 1,0 \\
\hline
\end{tabular}

Fonte: Elaborada pelos autores.

Em relação a ponderação para as categorias econômica, social e ambiental, todas foram classificadas com mesmo peso, por se entender que estas influenciam igualmente no processo de perdas de água.

\subsubsection{Matrizes de concordância e discordância}

A aplicação do método segue a partir do cálculo das matrizes de concordância e discordância. A matriz de concordância diz respeito à disposição do decisor em escolher a alternativa "i" em lugar de "j". Sendo i e j duas alternativas e k um critério.

- $i(k)$ é preferível a $j(k)$, sendo $i(k)>j(k)$

- $\mathrm{i}(\mathrm{k})$ é equivalente a $\mathrm{j}(\mathrm{k})$, sendo $\mathrm{i}(\mathrm{k})=\mathrm{j}(\mathrm{k})$

Lê-se i(k)>j(k) como: a alternativa "i" é preferível a "j" com relação ao critério k.

Para obtenção do valor de preferência, é calculada uma porcentagem ponderada dos critérios pelos quais a alternativa "i" é preferida da alternativa "j", através da Equação 5, sendo os resultados de cada aplicação da fórmula listados na matriz de concordância.

$c_{(l, k)}=\frac{\sum_{j} W^{+}+\frac{1}{2} W^{=}+\left(0,0 \cdot W^{-}\right)}{\sum_{j}{ }^{w_{j}}}$ 
Onde:

$\mathrm{c}(\mathrm{l}, \mathrm{k})=$ Grau de concordância entre as alternativas l e k.

$W^{+}=$peso do critério em que a alternativa é preferível.

$W^{=}=$peso do critério em que a alternativa se iguala a outra.

$W^{-}=$peso do critério em que a alternativa não é preferível, sendo esse multiplicado por zero;

$\mathrm{Wj}=$ todos os pesos.

Já o critério de discordância se refere a medição do desconforto causado pela escolha de "i" em relação a "j". Para seu cálculo é utilizado a Equação 6, sendo os resultados de cada aplicação da fórmula listados na matriz de discordância.

$D_{(i, j)}=\max \frac{[z(j, k)-z(i, k)]}{R}$

Onde:

$\mathrm{D}(\mathrm{i}, \mathrm{j})=$ Grau de discordância entre as alternativas i e j em relação ao critério k.

$\mathrm{Z}(\mathrm{j}, \mathrm{k})$ = avaliação a alternativa $\mathrm{j}$.

$Z(i, k)=$ avaliação a alternativa $\mathrm{i}$.

$\mathrm{R}=$ maior valor superior da escala numérica (de todos os critérios).

Para determinação dos valores a serem comparados nas matrizes de concordância e discordância foi utilizado a média dos valores normalizados dos indicadores selecionados para representar cada categoria do SAA.

A relação entre os valores de concordância e discordância é definida através dos umbrais de preferência (p) e o umbral de indiferença (q). Os valores de $\mathrm{p}$ e q foram definidos com base nas classificações estabelecidas por Cunha (2003), sendo 0,3 o valor do umbral de preferência e 0,2 o valor do umbral de indiferença. A partir desses valores foi feito o teste de dominância em que os valores das matrizes são comparados de acordo com o estabelecido na sentença da Equação 7.

$S k l=\left\{\begin{array}{c}1, \text { se } C(l, k)>\text { pe } D(i, j)<q \\ 0, \text { paraoutros valores }\end{array}\right.$

A partir das condições estabelecidas foi construída a matriz de veto de forma a deixar mais claro as condições atendidas. Essa matriz é montada de forma que quando a condição é satisfeita o local correspondente na matriz recebe o valor 1 , caso contrário esse é preenchido com o valor 0 .

A partir da matriz de veto, são analisadas as relações de sobreclassificação através da confecção de grafos. Como exposto por Almeida (2013), nesses grafos as situações são representadas a partir de setas em que o sentido da seta representa a sobreclassificação de uma alternativa por outra e a falta de setas representa incomparabilidade entre as alternativas.

Com a interpretação do grafo consegue-se distinguir qual a melhor e a pior alternativa a partir dos valores de sobreclassificação que cada alternativa possui, sendo a melhor delas a que possui o menor valor de sobreclassificiação, ou seja, a alternativa que não tem nenuma se sobrepondo a ela, logo sendo superada pela menor quantidade de alternativas; e a pior a que possui o maior valor de sobreclassificação, ou seja, a alternativa que tem o maior número de alternativas sobrepostas.

\section{RESULTADOS}

\subsection{Infraestrutura do sistema de abastecimento}

O SAA de Araruna - PB é integrado a outros municípios paraibano, atendendo aos municípios de Solânea, Bananeiras, Cacimba de Dentro, Riachão, Tacima, Damião e a própria Araruna, com água proveniente do reservatório Canafistula II. O sistema conta ainda com o Açude Jandaia para atender ao seu abastecimento, todavia, o sistema nunca o utilizou pois, desde a construção de seu sistema adutor, o corpo de água está seco.

De acordo com as informações obtidas pelo questionário aplicado na empresa prestadora do serviço, CAGEPA, o sistema de captação atual no Canafístula II é do tipo flutuante, permitindo a retirada da àgua na superfíce do manancial. O sistema de captação do Canafístula II conta com conjuntos motor-bomba e dois grupos de geradores de energia elétrica e tem uma capacidade de exploração de $82 \mathrm{~L} / \mathrm{s}$, transportados por adutoras de ferro fundido, com diâmetros entre $200 \mathrm{~mm}$ e 
$400 \mathrm{~mm}$ e extensão total de $27.549 \mathrm{~m}$. Para esse transporte é utilizado estação elevatória com bombas de $200 \mathrm{CV}$.

O tratamento é realizado em estação localizada no município de Cacimba de Dentro, com capacidade de tratar $64 \mathrm{~L} / \mathrm{s}$ de água em um esquema convencional. Após o tratamento, essa água é transportada em $16.700 \mathrm{~m}$ de adutora de ferro fundido, com diâmetro de $250 \mathrm{~mm}$, com auxílio de outra estação elevatório com bombas de $200 \mathrm{CV}$.

Para distribuição da água, é utilizado reservatório do tipo HINTRE, com capacidade de $500 \mathrm{~m}^{3}$ e rede de distribuição de PVC com diâmetros entre $50 \mathrm{~mm}$ a $250 \mathrm{~mm}$, totalizando $23 \mathrm{~km}$ de tubo.

\subsection{Perdas no sistema de abastecimento de água}

As perdas no SAA de Araruna-PB foram muito baixas devido aos longos períodos de suspensão do abastecimento, que tem como consequência baixas pressões e vazões. Na Figura 2, pode-se observar a série histórica das perdas por faturamento, perdas na distribuição, perdas lineares e perdas por ligação, podendo verificar a diminuição drástica dos valores de perdas a partir do ano de 2013, quando o abastecimento se tornou intermitente.

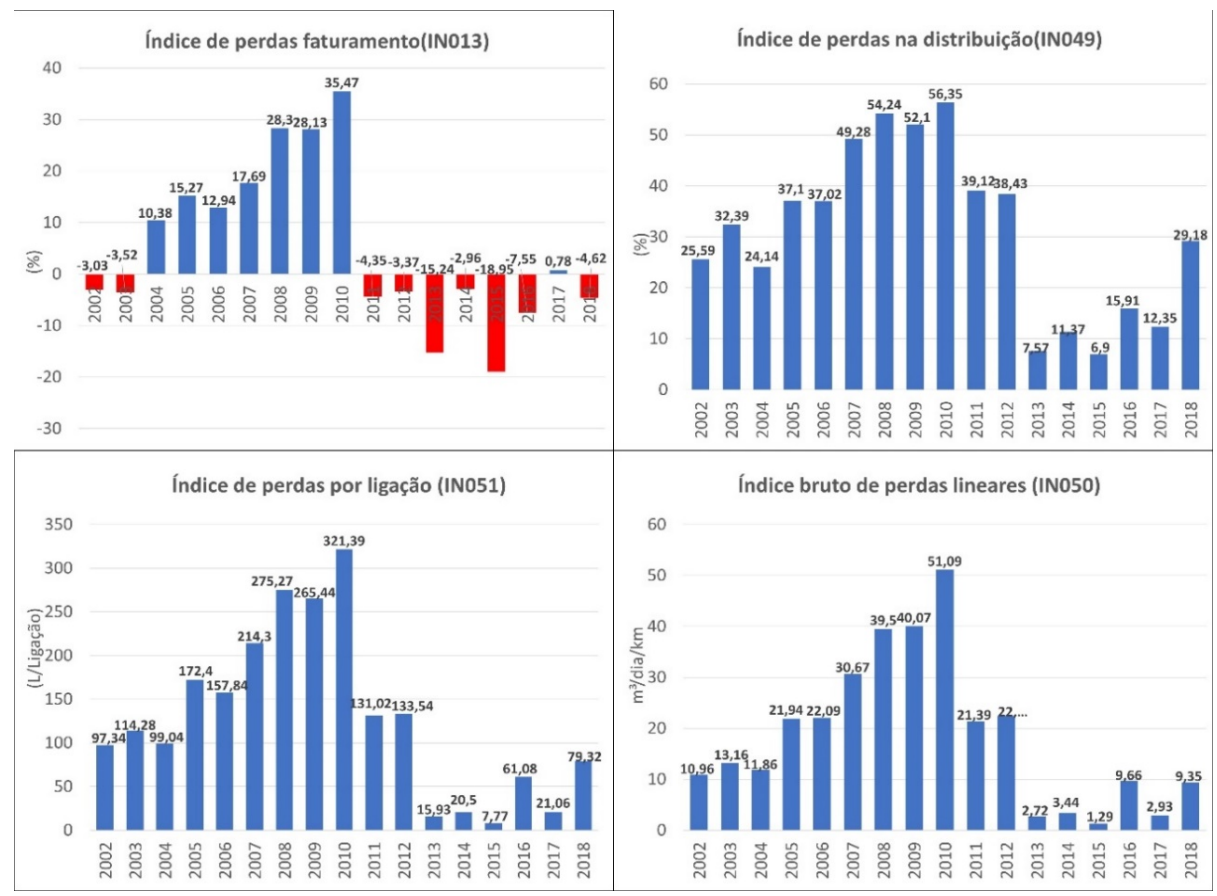

Figura 2 - Série histórica dos índices de perdas segundo o SNIS Fonte: Adaptada de Brasil (2018).

A sensibilidade dos indicadores de perdas à intermitência do sistema é atribuída ao fato da inexistência de indicadores perfeitos que consigam abranger as diversas situações que podem ocorrer nos sistemas. Existem diferentes relatos de problemas verificados na aplicação dos indicadores de perdas, dentre eles, a ocorrência de intermitência de abastecimento, como identificado no sistema em análise (Associação Brasileira de Engenharia Sanitária e Ambiental, 2015). Dados da Agência Executiva de Gestão das Águas na Paraíba (Agência Executiva de Gestão das Águas, 2021), com relação volume do açude Canafístula II nos ultimos 10 anos, mostram que a região passa por um problema crônico de escassez de água bruta desde de 2013. 0 abasteciemnto sofre intermitência devido aos níveis do reservatório, que opera somente com 9,5\% de seu volume total nos ultimos anos, em conformidade com informações da Companhia de Água e Esgotos da Paraíba.

Diante disso, como uma forma de aprimorar as análises referentes à situação das perdas no município, o SNIS poderia agregar em sua base de dados informações significativas, como a ocorrência de intermitências nos sistemas. Outra solução que pode ser utilizada é adotar um fator igual a $24 / \mathrm{n}$ o de horas com o sistema pressurizado para fazer a correção dos volumes medidos e, assim, simular condições próximas ao sistema integralmente pressurizado, de forma a não distorcer os números resultantes (Associação Brasileira de Engenharia Sanitária e Ambiental, 2015). 
Ao analisar o índice de perdas de faturamento é interessante verificar que seus valores sempre foram menores que as perdas na distribuição, o qual apresentou, inclusive, valores negativos em vários anos analisados. Isso pode indicar que o prestador de serviço faturou mais água do que efetivamente foi utilizado, provavelmente devido às cobranças de valores médios em residências sem micromedidor.

Por sua vez, as perdas na distribuição, que representam as perdas físicas do sistem, pois é calculada com base na água efetivamente consumida, chegoram a valores muito altos, de mais de 55\%. Segundo Charalambous (2011), altos valores de perdas na distribuição denotam um descuido na manutenção e operação desse sistema. Assim, para atingir baixos índices de perdas, deve-se melhorar a operação e manutenção, em concordância com a adequada prestação de serviços aos clientes.

Por outro lado, a partir do ano de 2013, o valor do índice de perda na distribuição teve uma baixa considerável para 7,57\%. No entanto, essa redução das perdas não pode ser atribuída a melhorias, já que não houve nenhum investimento no sistema no referido ano. Novamente, os baixos índices de perdas se justificam pela intermitência do abastecimento, visto que a redução da pressão que ocorre durante a intermitência do sistema está diretamente relacionada com a redução das perdas de água (Fundação Nacional de Saúde, 2014). De forma análoga, ocorreu com as perdas lineares e por ligação.

Todavia, o fornecimento intermitente não representa um método de gerenciamento eficiente de redes de distribuição, podendo em certos casos produzir efeitos adversos, como o aumento do número de tubos quebrados durante os períodos de intermitência e a contaminação da água da rede distribuição pela existência de pressão negativa. Mesmo que em certos casos, devido a exploração das fontes de água, a intermitência pareça ser a solução mais rápida e eficiente, o planejamento da distribuição e a conservação da rede podem ser soluções mais baratas e rápidas, garantindo a disponibilidade da água quando necessário (Charalambous, 2011).

É importante ressaltar que as perdas no SAA de Araruna - PB são essencialmente físicas, uma vez que o índice de micromedição é de 100\%, ou seja, todas as ligações de água possuem micromedidor.

\subsection{Análise do método multicriterial}

Seguindo os procedimentos metodológicos, foi realizada a coleta de dados do município de Araruna PB, utilizando as informações fornecidas pela CAGEPA ao banco de dados do SNIS no ano de 2018. Os indicadores já existentes foram coletados de forma direta, já o índice de investimento no SAA, que não é diretamente calculado pelo SNIS, teve o valor calculado com base nos dados apresentados no SNIS, segundo fórmula apresentada no Quadro 2. Os valores absolutos e normalizados dos indicadores são apresentados na Tabela 1.

Tabela 1 - Valores absolutos e normalizados dos indicadores

\begin{tabular}{|c|c|c|c|}
\hline Categoria & Indicadores & Valores absolutos & Valores normalizados \\
\hline \multirow{5}{*}{ Econômica } & E1 - Indicador de desempenho financeiro & 110,73 & 0,61 \\
\hline & E2 - Índice de evasão de receitas & 5,21 & 0,80 \\
\hline & E3 - Índice de produtividade & 3,21 & 0,19 \\
\hline & E4- Índice de faturamento de água & 104,62 & 1,00 \\
\hline & E5-Índice de investimento & 4,09 & 0,07 \\
\hline \multirow{3}{*}{ Social } & S1 - Índice de atendimento total de água & 40,26 & 0,00 \\
\hline & & & \\
\hline & $\begin{array}{l}\text { S2 - Volume de água disponibilizado por } \\
\text { economia }\end{array}$ & 8,50 & 0,00 \\
\hline \multirow{3}{*}{ Ambiental } & A1 - Consumo médio percapita de água & 57,8 & 1,00 \\
\hline & $\begin{array}{l}\text { A2 - Índice de micromedição relativo ao } \\
\text { consumo }\end{array}$ & 98,95 & 0,16 \\
\hline & A3 - Índice de macromedição & 0,00 & 0,00 \\
\hline
\end{tabular}

Fonte: Elaborada pelo autor.

Os conceitos de matriz de concordância e de discordância servem para ordenar as categorias estudadas comparadas com todas as outras categorias analisadas (Aires et al, 2013). A Tabela 2 apresenta os resultados para o cálculo do grau de concordância por meio da comparação par a par das categorias. Esses valores representam as relações de vantagem em que a categoria se sobressaiu da outra, tendo por base a média dos índices de referência estabelecidos variando de 0 a 1 , ou seja, mensura a vantagem relativa de cada categoria sobre todas as outras, na qual os valores de 
concordância deverão ser superiores ou iguais aos valores prestabelecidos e definem a máxima discordância tolerável.

Tabela 2 - Matriz de concordância

\begin{tabular}{c|c|c|c}
\hline \multicolumn{2}{c}{ Econômica } & Social & Ambiental \\
\hline Econômica & $\mathrm{X}$ & 0,49 & 0,49 \\
\hline Social & 0,00 & $\mathrm{X}$ & 0,00 \\
\hline Ambiental & 0,32 & 0,32 & $\mathrm{X}$ \\
\hline
\end{tabular}

Fonte: Elaborada pelos autores.

Quanto ao grau de discordância para as comparações par a par entre as categorias, tem-se os valores expostos na Tabela 3. Estes resultados expressam a quantidade de desconforto causado pela seleção de uma alternativa em relação a outra, ou seja, mensura a desvantagem relativa de cada categoria em relação sobre todas as outras.

Tabela 3 - Matriz de discordância

\begin{tabular}{c|c|c|c}
\hline \multicolumn{2}{c}{ Econômica } & Social & Ambiental \\
\hline Econômica & $\mathrm{X}$ & 0,00 & 0,00 \\
\hline Social & 0,53 & $\mathrm{X}$ & 0,42 \\
\hline Ambiental & 0,11 & 0,00 & $\mathrm{X}$ \\
\hline
\end{tabular}

Fonte: Elaborada pelos autores.

A relação das condições de preferência e desconforto de cada critério usando como parâmetro os umbrais de 0,3 para a matriz de concordância e 0,2 para a matriz de discordância, dá origem a matriz de veto, tendo os valores apresentados na Tabela 4.

Tabela 4 - Matriz de veto

\begin{tabular}{c|c|c|c}
\hline \multicolumn{2}{c}{ Econômica } & Social & Ambiental \\
\hline Econômica & $\mathrm{X}$ & 1,00 & 1,00 \\
\hline Social & 0,00 & $\mathrm{X}$ & 0,00 \\
\hline Ambiental & 0,00 & 1,00 & $\mathrm{X}$ \\
\hline
\end{tabular}

Fonte: Elaborada pelos autores.

A partir da matriz de veto é possível expor os resultados em forma de grafo de sobreclassificação, que mostra de forma mais objetiva quais as categorias apresentaram melhor desempenho em relação a outra, como exposto na Figura 3. As setas indicam a sobreposição do desempenho de uma categoria, na qual a seta se origina, em ralação a outra categoria, para qual a seta aponta. Desse modo, é possível vesualizar as categorias com mais sobreclassificações e as categorias que não tem sobreclassificação.

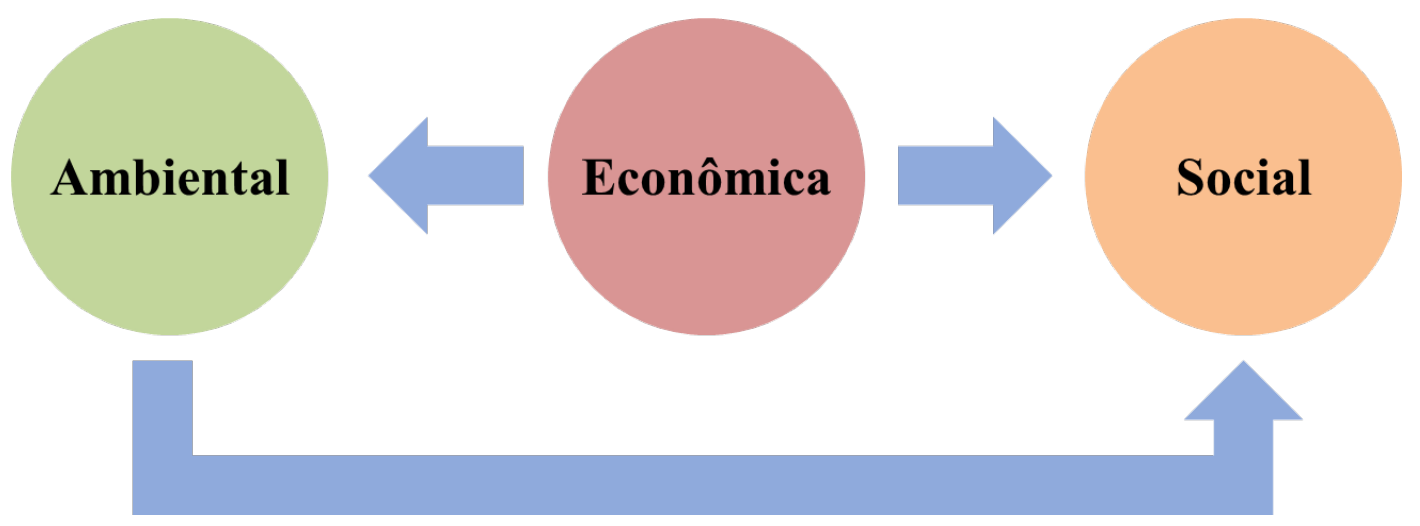

Figura 3 - Grafo de sobreclassificação Fonte: Elaborada pelos autores. 
Os valores de quantas vezes as setas se originam de uma categoria, condição de sobreclassificação, e quantas vezes elas apontam para a categoria, condição de subclassificação, são fornecidas na Tabela 5 a seguir.

Tabela 5 - Matriz de sobreclassificação

\begin{tabular}{c|c|c|c}
\hline Categoria & Sobreclassificação & Subclassificação & Ranque \\
\hline Econômica & 2 & 0 & $1^{\circ}$ \\
\hline Social & 0 & 2 & $3^{\circ}$ \\
\hline Ambiental & 1 & 1 & $2^{\circ}$ \\
\hline
\end{tabular}

Fonte: Elaborada pelos autores.

\subsection{Análise dos resultados}

Através do resultado obtido da aplicação do método ELECTRE II, foi verificado que a categoria social é a mais frágil do SAA do município de Araruna - PB, sendo a melhor área para investir ações prioritárias.

Entretanto, os problemas sociais causados por déficit no abastecimento de água vão além dos aspectos de atendimento e volume disponibilizado por economia, como analisado por esse estudo. A intermitência no abastecimento de água causa impactos na rotina domiciliar da população e ainda se aprofunda em questões de renda, onde, quanto maior a renda, menor o impacto nas rotinas domiciliares devido a maior a capacidade de armazenamento doméstico de água. Isso levanta questões de percepção de escassez, sendo que quanto menor a renda maior são as restrições feitas ao uso de água o que leva a naturalização dos impactos restritivos do racionamento (Del Grande et al., 2016). Se evidencia, também, que os baixos índices de perdas apresentados são devidos à baixa vazão e pressão, causadas pela intermitência do abastecimento de água.

Contudo, a experiência no cenário brasileiro geralmente aponta que o problema de perdas nas redes de abastecimento está relacionado aos vazamentos na rede, o que confronta com a análise global dos resultados desse estudo. A partir dessa divergência, é possível levantar a hipótese que o período de intermitência pode ter influenciado na sobreclassicação da categoria social. Logo, essa pesquisa norteia outra hipótese, para ser testada em trabalhos futuros, com a realização.

da análise das perdas sem a inclusão do período de intermitência da rede. Assim, deve ser iniciado ações de redução de perdas, especificamente perdas físicas, uma vez que todas as economias possuem micromedidores e o índice de perdas de faturamento é baixo. Devido à escassez de água no qual a região está sujeita em decorrência das características geográficas, a água disponível precisa ser aproveitada ao máximo, assim, quando houver reestabelecimento do abastecimento de água, devem ser adotadas medidas de controle de perdas, para evitar desperdícios (Cambrainha \& Fontana, 2015).

Apesar da indicação prioritária de investimento na categoria social, é possível verificar pelos valores dos indicadores selecionados que as categorias ambiental e econômica também necessitam de atenção. Nesse panorama, o combate as perdas de água diminuem a evasão econômica das operadoras, bem como permitem que mais pessoas sejam atendidas sem necessitar aumentar a exploração do recurso.

Ao analisar o impacto ambiental causado pelas perdas de água, verifica-se um agravamento das questões ambientais, principalmente em regiões que passam por situações de escassez hídrica. Esse agravamento ocorre devido ao cenário de perdas fazer com que haja a necessidade de que maiores volumes de água sejam retirados da natureza para encobrir a ineficiência, vazamentos e outros problemas na distribuição de água do sistema de abastecimento. Assim, com a melhoria da eficiência e com redução das perdas, seria possível atender um maior número de pessoas, conseguindo levar água potável a quem não tem, com a mesma disponibilidade hídrica (Instituto Trata Brasil, 2013).

Em relação a categoria econômica, os baixos investimentos em manutenção dos sistemas de abastecimento, unidos a má gestão e tarifação inadequada, tem levado a dificuldades dos prestadores de serviços viabilizar a sustentabilidade econômica dos serviços de abastecimento de água. Uma das formas de causar melhorias econômicas no SAA seria a adoção de medidas de redução de perdas, de modo que essas alcancem um percentual de $20 \%$, além de causar melhoras econômicas no sistema o alcance desse cenário poderia reduzir as tarifas do serviço em índices de 25\% (Instituto Trata Brasil, 2013).

No entanto, o que tem se verificado é que regiões como o nordeste que sofre de grande dependência climática para garantir o abastecimento, os prestadores de serviço têm investido 
principalmente em obras para aumentar a captação de água. Porém o cenário de perdas dessa região, que apresenta altos índices, indica que a gestão e redução de perdas deveria ser tratada como objetivo principal para investimento (Cambrainha \& Fontana, 2015).

Por outro lado, o aumento da preocupação com as decisões envolvendo o saneamento, provocou também um aumento na procura por ferramentas e métodos que auxiliem no progresso da área sanitária, de modo a diminuir a subjetividade das decisões (Goffi et al., 2018). Mesmo o cenário do saneamento envolvendo um grande número de variáveis, a metodologia de análise multicritério confirmou ser uma importante ferramenta, conseguindo reduzir as incertezas e auxiliar na estruturação do problema de forma objetiva e organizada (Karimi et al., 2011; Maurer et al., 2012; De Paula, 2013).

A estruturação do problema permite que o gestor, durante a tomada de decisão, compare as alternativas de maneira simultânea, permitindo eficiência no planejamento e a seleção da melhor alternativa. Ainda, é importante destacar que esse modelo possui grande abrangência, podendo ser aplicada para simplificação de cenários de diversas áreas, desde que a essência principal do modelo seja mantida (Goffi et al., 2018).

Apesar da eficiência ser comprovada, é muito importante saber definir se a quantidade de parâmetros é suficiente para descrever o cenário, além de ter a sensibilidade da veracidade dos dados utilizados, antes de considerá-los. Visto que nem sempre o modelo retrata a abrangência de toda a realidade, assim o conhecimento aprofundado do local de estudo permite saber apontar as nuances que venham a alterar drasticamente os resultados finais (Albuquerque, 2018).

\section{CONCLUSÕES}

Dificuldades com o abastecimento de água em municípios é um grave problema na vida da população urbana, principalmente quando é um problema crônico, como no caso do municipio de Araruna - PB, que há anos vem sofrendo com a intermitência do abastecimento devido à escassez de água bruta na região. Neste sentido, a vista de que a má gestão dos recursos hídricos incapacitou o acesso a água, é necessário investir na gestão da demanda, para tornar os usos mais eficientes, podendo agir entre outras vertentes na diminuição de perdas de água.

Diante disso, o trabalho e a aplicação da metodologia multicriterial se mostrou bastante pertinente para buscar soluções racionais ao problema das perdas de água, indicando quais as melhores formas de otimizar o SAA de Araruna - PB. Com a análise do sistema de abastecimento de água estudado, verificou-se que as perdas de água são pequenas, não devido a eficiência do sistema, mas pela intermitência no abastecimento, visto que se há pouca água distribuída, pouca água é perdida.

Ao analisar esse cenário através do método multicritério de apoio a tomada decisão ELECTRE II, foi verificado que a categoria social é a que possui mais fragilidade, influenciando negativamente as perdas de água. Assim, a solução aponta que os problemas listados na categoria social referentes ao atendimento total de água e ao volume de água disponibilizado por economia acabam influenciando de forma mais crítica os problemas com as perdas, pois a pouca quantidade de água e baixa pressão fornecida diminui as perdas no sistema de abastecimento.

Mesmo a solução apontando a categoria social como a principal influência negativa para as perdas de água, as categorias ambiental e econômica devem ser levadas em consideração para minimização do problema ligado as perdas, uma vez que a disponibilidade hídrica e os recursos financeiros necessários à otimização do sistema estão comprometidos.

Vale a pena ressaltar que alcançar um cenário de um SAA sem perdas é impossível, pois mesmo os sistemas que apresentam as condições econômicas, sociais e ambientais adequadas apresentam perdas, uma vez que se torna inviável economicamente alcançar perdas nula. No entanto, a busca pela minimização desse problema se mostra importante para que o sistema funcione da melhor forma e, com isso, poder minimizar os efeitos provocados pelos constantes racionamentos de água que passam a população local.

\section{REFERÊNCIAS BIBLIOGRÁFICAS}

Adger, W. N. (2006). Vulnerability. Global Environmental Change, 16(3), 268-281.

http://dx.doi.org/10.1016/j.gloenvcha.2006.02.006 
Agência Executiva de Gestão das Águas - AESA. (2021). Monitoramento últimos volumes informados dos açudes. Recuperado em 17 de julho de 2021, de http://www.aesa.pb.gov.br/aesa-website/monitoramento/volumeacude/?id_acude=3036

Aires, R. F. F., Braga, L. G., Silveira Neto, J. C., Valentim Neto, A. J., \& Araújo, A. G. (2013). Apoio à decisão de compra na indústria de cimentos e derivados: um estudo Utilizando o Método ELECTRE. In Anais do IV Encontro de Administração da Informação. Bento Gonçalvez, RS.

Albuquerque, A. M., Ferreira, Y. B., Silva, S. B., \& Sales, M. C. L. (2019). Balanço hídrico como ferramenta de gerenciamento de recursos hídricos: aplicação na área de influência direta do Açude Castanhão-CE. Revista da Casa da Geografia de Sobral, 21(2), 454-466. http://dx.doi.org/10.35701/rcgs.v21n2.601

Albuquerque, B. A. (2018). Modelo utilizando sistema de informações geográfica para análise multicritério de redes de distribuição de água. In Anais do $29^{\circ}$ Congresso Nacional de Saneamento e Meio Ambiente (pp. 1-16). São Paulo: AESABESP.

Albuquerque, T. M. A., Ribeiro, M. M. R., \& Vieira, Z. M. C. L. (2008). Análise multicriterial de alternativas tecnológicas para redução do consumo de água. Revista Brasileira de Recursos Hídricos, 13(4), 99-110. http://dx.doi.org/10.21168/rbrh.v13n4.p99-110

Almeida, A. T. (Org.). (2013). Processo de decisão nas organizações: construindo modelos de decisão multi-critério. São Paulo: Atlas.

Associação Brasileira de Engenharia Sanitária e Ambiental - ABES. (2015). Controle e redução de perdas nos sistemas públicos de abastecimento de água posicionamento e contribuições técnicas da ABES. Recuperado em 25 de julho de 2019, de http://abes-dn.org.br/pdf/28Cbesa/Perdas_Abes.pdf

Biasutti, S., \& Coelho, E. R. C. (2019). Normatização de indicadores de perdas de água: a experiência das agências reguladoras no Brasil. Revista DAE, 215(67), 17-24.

Boruff, B., Biggs, E., Pauli, N., Callow, N., \& Clifton, J. (2018). Changing water system vulnerability in Western Australia's Wheatbelt region. Applied Geography (Sevenoaks, England), 91, 131-143. http://dx.doi.org/10.1016/j.apgeog.2017.12.016

Brasil. Sistema Nacional de Informações sobre Saneamento - SNIS. (2018). 24o Diagnóstico dos Serviços de Água e Esgotos - 2018. Recuperado em 25 abril de 2019, de http://www.snis.gov.br/diagnostico-anual-agua-eesgotos/diagnostico-dos-servicos-de-agua-e-esgotos-2018

Cambrainha, G. M. G., \& Fontana, M. E. (2015). Análise da aplicação de investimentos em perdas de água no nordeste brasileiro. Revista Eletrônica em Gestão, Educação e Tecnologia Ambiental, 19(2), 983-994. http://dx.doi.org/105902/2236117015520.

Charalambous, B. (2011). Water supply networks and pipelines: The Hidden Costs of Resorting to Intermittent Supplies. Recuperado em 25 abril de 2019, de https://warrington.ufl.edu/public-utility-researchcenter/wp-content/uploads/sites/57/2018/02/water-supply-networks-pipelines-hidden-cost-resortingintermittent-supplies-2011-12.pdf

Colombo, A. F., \& Karney, B. W. (2005). Impacts of leaks on energy consumption in pumped systems with storage. Journal of Water Resources Planning and Management, 131(2), 146-155. http://dx.doi.org/10.1061/(ASCE)0733-9496(2005)131:2(146)

Cunha, M. J. B. (2003). Aplicação da metodologia ELECTRE I de apoio da decisão multicritério na priorização de transporte de mercadoria. In Anais do XXIII Anais do Encontro Nacional de Engenharia de Produção. Ouro Preto: ABEPRO.

De Paula, R. L. (2013). Metodologia para avaliação de desempenho operacional de tratamento de esgotos, utilizando métodos multiobjectivo e indicadores (Dissertação de Mestrado). Universidade de Brasília, Brasília.

Del Grande, M. H., Galvão, C. O., Miranda, L. I. B., \& Sobrinho, L. D. G. (2016). A percepção de usuários sobre os impactos do racionamento de água em suas rotinas domiciliares. Ambiente \& Sociedade, 19(1), 165-184. http://dx.doi.org/10.1590/18094422asoc150155r1v1912016

Fundação Nacional de Saúde - FUNASA. (2014). Redução de perdas em sistemas de abastecimento de água. Brasília: Ministério da Saúde. Recuperado em 25 de abril de 2019, de <http://www.funasa.gov.br/site/wpcontent/files_mf/reducao_de_perdas_em_saa74.pdf.>

Gnadlinger, J. (2015). Água de chuva no manejo integrado dos recursos hídricos em localidades semiáridas: Aspectos históricos, biofísicos, técnicos, econômicos e sociopolíticos. In D. B. Santos, S. S. Medeiros, L. T. L. Brito, J. Gnadlinger, E. Cohim, V. P. S. Paz \& H. R. Gheyi (Orgs.), Captação, manejo e uso de água de chuva (pp. 1-460). Campina Grande: INSA. 
Goffi, A. S., Bortoluzzi, S. C., Trojan, F., \& Goffi, A. (2018). Abordagem multicritério na seleção de alternativas de tratamento de efluentes: uma revisão sistêmica da literatura. Holos Environment, 18(1), 28-43. http://dx.doi.org/10.14295/holos.v18i1.12246

Gomes Júnior, S. F., Chaves, M. C. C., Pereira, E. R., \& Mello, J. C. B. (2008). Método multicritério electre ii na avaliação de pilotos no campeonato de Fórmula 1. SPOLM.

Govindan, K., \& Jepsen, M. (2016). ELECTRE: a comprehensive literature review on methodologies and applications. European Journal of Operational Research, 250(1), 1-29. http://dx.doi.org/10.1016/j.ejor.2015.07.019

Guedes, M., Ribeiro, M., \& Vieira, Z. (2014). Alternativas de gerenciamento da demanda de água na escala de uma cidade. Revista Brasileira de Recursos Hídricos, 19(2), 123-134. http://dx.doi.org/10.21168/rbrh.v19n2.p123-134

Gupta, A., Bokde, N., Marathe, D., \& Kulat, K. (2017). Leakage reduction in water distribution systems with efficient placement and control of pressure reducing valves using soft computing techniques. Engineering, Technology \&. Applied Scientific Research, 7(2), 1528-1534.

Instituto Trata Brasil - ITB. (2013). Perdas de água: Entraves ao avanço do saneamento básico e riscos de agravamento à escassez hídrica no Brasil. Recuperado em 13 de maio de 2019, de http://www.tratabrasil.org.br/datafiles/uploads/perdas-de-agua/estudo-completo.pdf

Instituto Trata Brasil - ITB. (2018). Perdas de água 2018 (SNIS 2016): desafios para disponibilidade hídrica e avanço da eficiência do saneamento básico. Recuperado em 17 de julho de 2021, de http://www.tratabrasil.org.br/images/estudos/itb/perdas-2018/estudo-completo.pdf

Instituto Trata Brasil. (2015). Perdas de água: novo estudo mostra as perdas nos sistemas de distribuição, a baixa evolução desses indicadores e os grandes desafios para a solução. Recuperado em 13 de maio de 2021, de http://www.tratabrasil.org.br/perdas-de-agua-desafios-ao-avancodo-saneamento-basico-e-aescassez-hidrica-2

Ishizaka, A., \& Nemery, P. (Orgs.). (2013). Multi-criteria decision analysis: methods and softwares. Nova Jersey: Wiley.

Karimi, A. R., Mehrdadi, N., Hashemian, S. J., Nabi, B G. R., \& Tavakkoli M. R. (2011). Selection of wastewater treatment process based on the analytical hierarchy process and fuzzy analytical hierarchy process methods. International Journal of Environmental Science and Technology, 8, 267-280. http://dx.doi.org/10.1007/BF03326215

Kouchi, D. H., Esmaili, K., Faridhosseini, A., Sanaeinejad, S. H., Khalili, D., \& Abbaspour, K. C. (2017). Sensitivi-ty of calibrated parameters and water resource estimates on different objective functions and optimization algorithms. Water (Basel), 9(6), 384-400. http://dx.doi.org/10.3390/w9060384

Maurer, M., Bufardi, A., Tilley, E., Zurbrügg, C., \& Truffer, B. A. (2012). Compatibility-based procedure de-signed to generate potential sanitation system alternatives. Journal of Environmental Management, 104, 51-61. http://dx.doi.org/10.1016/j.jenvman.2012.03.023

Mota, C. M. M., \& Almeida, A. T. (2007). Método multicritério ELECTRE IV-H para priorização de atividades em projetos. Pesquisa Operacional, 27(2), 247-269.

Neves, Y. T. (2016). Tratamento alternativo de águas subterrâneas em comunidades rurais (Trabalho de Conclusão de Curso). Curso de Engenharia Civil, Universidade Estadual da Paraíba, Araruna.

Nunes, L. G. C. F., Soares, A. E. P., Silva, J. K., França, F. F., \& Silva, S. R. (2020). Educação para conservação de águas em escolas públicas: relato de experiência em Pernambuco. Revista Extensão em Foco, 20, 102-114. http://dx.doi.org/10.5380/ef.v0i20.67216

Ogata, I. S. (2014). Desenvolvimento do índice de pobreza hídrica para a bacia hidrográfica do Rio Paraíba (Dissertação de mestrado). Universidade Federal de Campina Grande, Campina Grande.

Oliveira, M., Melo, E., Moser, D., \& Amaro, R. (2013). Apoio a decisão em sistemas de produção: um estudo de caso na construção naval. In Anais do XXXIII Encontro Nacional de Engenharia de Produção (pp. 1-15). Salvador: ABREPRO.

Pompermayer, R. S., Paula Júnior, D. R., \& Cordeiro Netto, O. M. (2007). Análise Multicritério como Instrumento de Gestão de Recursos Hídricos: o caso das bacias dos Rios Piracicaba, Capivari e Jundiaí. Revista Brasileira de Recursos Hídricos, 12(3), 17-127. http://dx.doi.org/10.21168/rbrh.v12n3.p117-127

Silva, A. C. N. (2019). Panorama de perdas em sistemas de abastecimentos de água no Brasil (Trabalho de Conclusão de Curso). Universidade Estadual da Paraiba, Campina Grande. 
Szajubok, N. K., Mota, C. M. M., \& Almeira, A. T. (2006). Uso do método multicritério ELECTRE TRI para classificação de estoques na construção civil. Pesquisa Operacional, 26(3).

Turner, B. L., Kasperson, R. E., Matson, P. A., Mccarthy, J. J., Corell, R. W., Christensen, L., Eckley, N., Kasper-Son, J. X., Luers, A., Artello, M. L., Polsky, C., Pulsipher, A., \& Shiller, A. (2003). A framework for vulnerability analysis in sustainability science. Proceedings of the National Academy of Sciences of the United States of America, 100(14), 8074-8079. http://dx.doi.org/10.1073/pnas.1231335100

Venturini, M. A. A. G. (2003). Metodologia de análise e decisão multicriterial para a reabilitação de sistemas de abastecimento de água (Tese de doutorado). Universidade Estadual de Campinas, Campinas.

\section{Contribuições dos autores:}

Cinthia Maria de Abreu Claudino: Coleta de dados Tabulação, Análise estatística dos dados Criação de tabelas e figuras Redação do texto, Padronização das normas de acordo com a revista Revisão do texto

Vanine Elane Menezes de Farias: Tabulação, Análise estatística dos dados Redação do texto, Padronização das normas de acordo com a revista.

Alessandra Gomes Brandão: Criação de tabelas e figuras Redação do texto, Revisão do texto.

Igor Souza Ogata: Coleta de dados, Análise estatística dos dados Criação de tabelas e figuras Redação do texto e Revisão do texto

Rivaildo da Silva Ramos Filho: Coleta de dados, Análise estatística dos dados e Redação do texto.

Thiago de Sá Sena: Revisão do texto Redação do texto e Criação de tabelas e figuras. 